\title{
Transverse Ridge of Premolars and Oblique Ridge of Maxillary Molars are in Divine Golden Ratio
}

\author{
Sachin C Sarode ${ }^{1}$, Nikunj Maniyar ${ }^{2}$, Gargi S Sarode ${ }^{3}$, Sujata Yerwadekar ${ }^{4}$, Rajesh Shetty ${ }^{5}$, Amol R Gadbail $^{6}$,
} Shailesh Gondivkar ${ }^{7}$, Rahul Anand ${ }^{8}$, Shankargouda Patil ${ }^{9}$

\begin{abstract}
Aims: In premolars and molars, transverse ridge (TR) and oblique ridge (OR) play major roles in providing strength and stability to the cusps in particular and tooth as a whole. Hence, they could be divided at the central groove or the transverse groove in a divine golden ratio.

Materials and methods: Twenty pairs of maxillary and mandibular casts were retrieved from the Department of Orthodontics. The lengths of buccal triangular ridge (BTR), lingual triangular ridge (LTR) and TR of maxillary and mandibular first and second premolars were measured. Similarly, lengths of distal cuspal ridge of mesiopalatal cusp, triangular ridge of distobuccal cusp (DBTR) and OR of the maxillary first and second molars were measured using a brass wire and a Vernier caliper. The TR:BTR, BTR:LTR, OR:distal ridge of mesiopalatal cusp (MPDR), and MPDR:DBTR ratios were calculated to find out the possibility of golden ratio.

Results: Transverse ridges of the premolars and OR of the maxillary first and second molars were divided at the central groove in a divine golden ratio. The mean TR/BTR ratio for maxillary first premolar was $1.61 \pm 0.007$, whereas the mean BTR/LTR ratio was $1.63 \pm 0.02$. The mean TR/BTR ratio for maxillary second premolar was $1.61 \pm 0.01$, whereas the mean BTR/LTR ratio was $1.62 \pm 0.04$. The TR/BTR and BTR/LTR ratios for mandibular first premolar were $1.6 \pm 0.008$ and $1.64 \pm 0.02$, respectively. Similarly, the mean TR/BTR ratio for mandibular second premolar was $1.61 \pm 0.01$, whereas the mean BTR/LTR ratio was $1.63 \pm 0.02$.

Conclusion: The ORs of maxillary first molar ridge and TR of maxillary and mandibular premolars are divided into two parts at transverse and central grooves, respectively, in a divine ratio.

Clinical relevance: The ORs of maxillary first molar ridge and TR of maxillary and mandibular premolars are divided into two parts at transverse and central grooves, respectively, in a divine ratio. This signifies the strength and stability of the occlusal table in the posterior teeth, as the golden ratio is the mark of functional excellence. Golden ratio should be taken into consideration while restoration or fabrication of the posterior teeth.

Keywords: Golden ratio, Maxillary molars, Oblique ridge, Premolars, Tooth morphology, Transverse ridge.

The Journal of Contemporary Dental Practice (2020): 10.5005/jp-journals-10024-2783
\end{abstract}

\section{INTRODUCTION}

The golden ratio or the divine ratio as they say is omnipresent and has been an area of extensive research in not only dentistry but also several other disciplines. Although widely observed in almost every aspect of nature, origin and first use of the golden ratio by mankind remain conceal and enigmatic. Formulated as one of the Euclid's elements, the golden ratio was used extensively in the Greek architecture such as the Parthenon on the Acropolis of Athens and has been employed in the field of art since years now. ${ }^{1}$ Euclid 2 is considered as one of the pioneers who demonstrated the method of dividing a straight line by means of the golden proportion; Kepler called it the "Divine proportion." ${ }^{2,3}$ In Euclid's words: "A straight line is said to have been cut in extreme and mean ratio when, as the whole line is to the grater segment, so is the greater to the lesser." ${ }^{\prime 4}$

This divine ratio which is better known by the term "Phi" $(\varphi)$, from the Greek sculptor Phidias who extensively studied it, corresponds to the ratio of $1: 1.618 .^{2}$ It is found not only in geometrical elements but also in living subjects including the human body. Years ago Marcus Pollio suggested that the height of a well-proportioned man is equal to the length of his outstretched arms. The body and outstretched arms can be inscribed in a square, whereas the hands and feet are emblazoned in a circle. With such organization, the human body can be divided into two parts at the naval that are represented in the proportion of the golden rectangle or in the divine proportion. ${ }^{5}$ Nature is abundant with examples of golden proportion which suggests that the golden ratio is a law of

\footnotetext{
${ }^{1-3}$ Department of Oral Pathology and Microbiology, Dr DY Patil Dental College and Hospital, Dr DY Patil Vidyapeeth, Pune, Maharashtra, India ${ }^{4}$ Department of Orthodontics, Dr DY Patil Dental College and Hospital, Dr DY Patil Vidyapeeth, Pune, Maharashtra, India

${ }^{5}$ Department of Conservative Dentistry and Endodontics, Dr DY Patil Dental College and Hospital, Dr DY Patil Vidyapeeth, Pune, Maharashtra, India

${ }^{6}$ Department of Dentistry, Indira Gandhi Government Medical College and Hospital, Nagpur, Maharashtra, India

${ }^{7}$ Department of Oral Medicine and Radiology, Government Dental College and Hospital, Nagpur, Maharashtra, India

${ }^{8}$ Department of Oral Pathology and Microbiology, Government Dental College and Hospital, Rohtak, Haryana, India

${ }^{9}$ Department of Maxillofacial Surgery and Diagnostic Sciences, Division of Oral Pathology, College of Dentistry, Jazan University, Jazan, Kingdom of Saudi Arabia

Corresponding Author: Sachin C Sarode, Department of Oral Pathology and Microbiology, Dr DY Patil Dental College and Hospital, Dr DY Patil Vidyapeeth, Pune, Maharashtra, India, Phone: +91 9922491465, e-mail: drsachinsarode@gmail.com

How to cite this article: Sarode SC, Maniyar N, Sarode GS, et al. Transverse Ridge of Premolars and Oblique Ridge of Maxillary Molars are in Divine Golden Ratio. J Contemp Dent Pract 2020;21(5):486-489. Source of support: Nil

Conflict of interest: None
}

(-) The Author(s). 2020 Open Access This article is distributed under the terms of the Creative Commons Attribution 4.0 International License (https://creativecommons. org/licenses/by-nc/4.0/), which permits unrestricted use, distribution, and non-commercial reproduction in any medium, provided you give appropriate credit to the original author(s) and the source, provide a link to the Creative Commons license, and indicate if changes were made. The Creative Commons Public Domain Dedication waiver (http://creativecommons.org/publicdomain/zero/1.0/) applies to the data made available in this article, unless otherwise stated. 
nature that is most widely observed and acknowledged across the globe. Apart from being esthetically pleasing, the dimensions of golden ratio are functionally the most efficient. It has been found to be present everywhere, from Egyptian pyramids to the great Parthenon to human DNA and studies that explore this startling ratio are plentiful in the literature. ${ }^{6}$

The emergence of several points with regard to golden proportion in human body and body parts has increased the interest of not only anatomists, scientists, and artists but also of dentists and esthetes. Many studies have been carried out till date to unveil the presence of divine proportion in different aspects of dentistry. Levin ${ }^{3}$ observed that the width of maxillary central incisor is in golden proportion with the width of maxillary lateral incisor and that of lateral incisor with canine. Similarly, the ratio of height to width of the face is also found to be in golden proportion. ${ }^{4}$ Anand et al. ${ }^{6}$ demonstrated that the human permanent teeth are divided into two parts at the cementoenamel junction in the divine ratio. Teeth are considered as the most important structures in the oral cavity, both esthetically and functionally. From providing appealing smiles to the function of mastication and load bearing, their relevance is multidisciplinary. Keeping this in mind, we strongly believed that some of the functional aspects of human teeth should follow the divine rule of golden proportion.

In premolars and molars, triangular ridge plays a major role in providing strength and stability to the cusps in particular and tooth as a whole. Hence, we hypothesized that transverse ridge (TR) and oblique ridge (OR) should be divided at some unknown point in the golden ratio. After careful analysis, we identified the central groove (for TR of premolars) and the transverse groove (for OR of maxillary molars) as the separation landmark for the golden ratio. With this view in mind, we measured the buccal and lingual/palatal triangular ridges of maxillary and mandibular first and second premolars with the dividing point at the central groove and the OR of maxillary first and second molars with the dividing line at the transverse groove and tried to approximate the results to the divine golden ratio.

\section{Materials and Methods}

\section{Study Sample}

A total of 20 orthodontic patient casts (age range: 18-25 years) were collected and segregated for the analysis. Diagnostic casts of only those patients with natural permanent teeth having well-defined anatomic elements and without any regressive alterations were included in the study sample. Casts that were broken, attrited, abraded, eroded, or lacked peculiar anatomic details were excluded from the study. No casts with the presence of fixed dental prosthesis on posterior teeth were considered for the study. For mandibular second premolars, only casts with " $\mathrm{H}$ " type of groove were selected for the analysis.

In total, 20 teeth each for maxillary first premolar, maxillary second premolar, mandibular first premolar, mandibular second premolar, maxillary first molar and maxillary second molar from 20 casts were finally analyzed for the presence of golden ratio in ORs and TRs. Either right side or left side tooth was selected randomly for the analysis.

\section{Calculating the Golden Ratio Maxillary and Mandibular Premolars}

The lengths of buccal triangular ridge (BTR), lingual triangular ridge (LTR) and TR, which is nothing but the total length of BTR and LTR, of all the maxillary and mandibular first and second premolars were measured and two ratios were calculated: the TR length to the BTR length (TR:BTR) and the BTR length to the LTR length (BTR:LTR). These ratios were then allied to check the approximation for golden ratio using the following formula.

$\frac{\text { Transverse ridge length }}{\text { Buccal triangular ridge length }}=\frac{\text { Buccal triangular ridge length }}{\text { Lingual triangular ridge length }}$

\section{Maxillary First Molars}

The lengths of distal cuspal ridge of mesiopalatal cusp (MPDCR), triangular ridge of distobuccal cusp (DBTR) and OR, which is the sum of distal ridge of mesiopalatal cusp (MPDR) and DBTR, of all the maxillary first and second molars were measured and two ratios were calculated: the OR to MPDR (OR:MPDR) and MPDR to DBTR (MPDR:DBTR). These ratios were then allied to check the approximation for the golden ratio using the following formula.

$\frac{\text { Oblique ridge }}{\text { Distal cusp ridge of mesiopalatal cusp }}$
$\frac{\text { Distal cusp ridge of mesiopalatal cusp }}{\text { Triangular ridge of distobuccal cusp }}$

\section{Measurement of Ridge Lengths}

A brass wire was used to measure the lengths of BTR and LTR of all the premolars. The brass wire was first adapted along the BTR of the buccal cusp from the cusp tip to the central groove and then the length was measured using the vernier caliper. Similarly, for determining the length of LTR the wire was adapted along the LTR of the lingual cusp from the cusp tip to central groove and the length was measured (Fig. 1). The length of TR was calculated by summing the BTR length and the LTR length. The length of OR of maxillary first and second molars was calculated in a similar fashion using the brass wire and vernier caliper. The brass wire was adapted along the MPDR from the cusp tip to transverse groove and along the DBTR from the cusp tip to transverse groove (Fig. 2). The length of OR was calculated by adding the distal cuspal ridge length of mesiopalatal cusp and the triangular ridge length of distobuccal cusp.

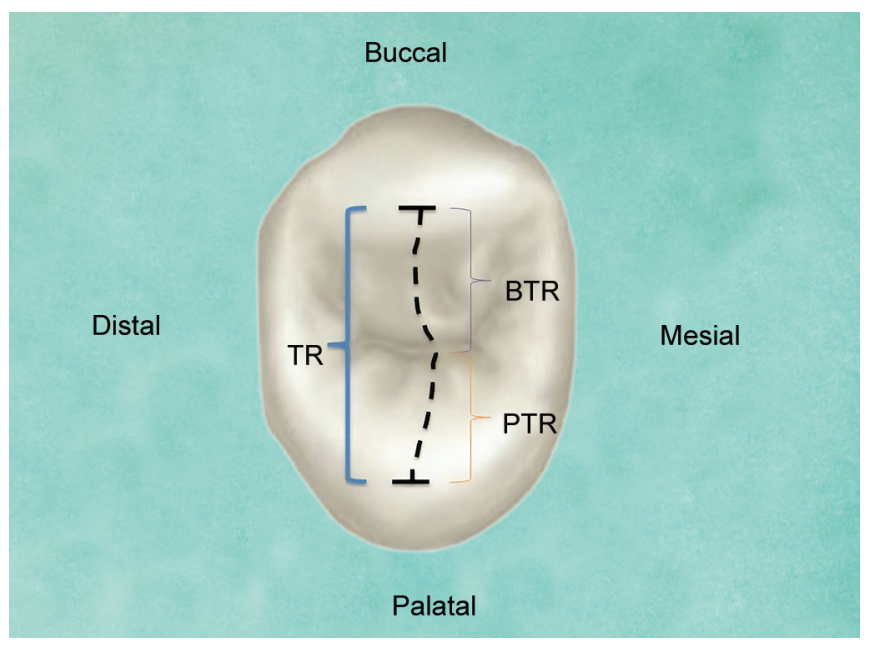

Fig. 1: Schematic presentation of premolar showing the calculation of lengths of buccal triangular ridge (BTR), lingual triangular ridge (LTR) and transverse ridge (TR) 


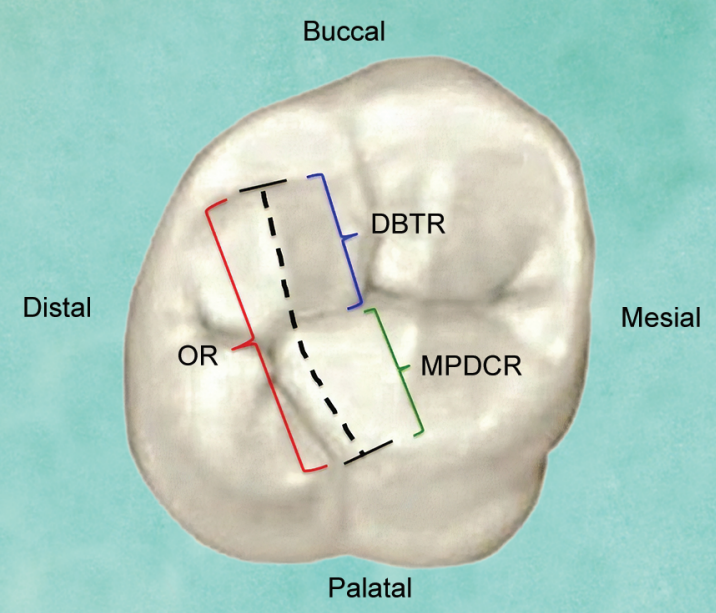

Fig. 2: Schematic presentation of molar showing the calculation of lengths of triangular ridge of distobuccal cusp (DBTR), distal cuspal ridge of mesiopalatal cusp (MPDCR) and oblique ridge (OR)

\section{Statistical Analyses}

All statistical analyses were carried out using software (IBM SPSS Statistics Inc.) version 20 (SPSS, Chicago, IL, USA). Mean and standard deviation of golden ratio were calculated for each group. Comparison of the golden ratio obtained from two different methodologies was done by the unpaired " $t$ " test.

\section{Results}

Transverse ridges of all the premolars and OR of all the maxillary first and second molars were divided at the central groove in a divine golden ratio.

The mean BTR, LTR and TR for maxillary first premolar were $4.07 \pm$ $0.64,2.49 \pm 0.4$ and $6.56 \pm 1.04$, respectively. The mean TR/BTR ratio for maxillary first premolar was $1.61 \pm 0.007$, whereas the mean $B T R / L T R$ ratio was $1.63 \pm 0.02$. There was statistically significant difference between TR/BTR and BTR/LTR $(p<0.00001)$. Similarly, the mean BTR, LTR and TR for maxillary second premolar were $4.07 \pm$ $0.52,2.5 \pm 0.32$ and $6.57 \pm 0.84$, respectively. The mean TR/BTR ratio for maxillary second premolar was $1.61 \pm 0.01$, whereas the mean BTR/LTR ratio was $1.62 \pm 0.04$ with statistically insignificant differences between the two $(p<0.18)$.

In the case of mandibular first premolar, mean BTR, LTR and TR were $2.72 \pm 0.31,1.65 \pm 0.18$ and $4.37 \pm 0.5$, respectively. The TR/BTR and BTR/LTR ratios were $1.6 \pm 0.008$ and $1.64 \pm 0.02$, respectively, with statistically significant differences among them $(p<0.00001)$. Similarly, the mean BTR, LTR and TR for mandibular second premolar were $3.44 \pm 0.52,2.16 \pm 0.29$ and $5.69 \pm 0.73$, respectively. The mean TR/BTR ratio for mandibular second premolar was $1.61 \pm$ 0.01 , whereas the mean BTR/LTR ratio was $1.63 \pm 0.02$. There was statistically significant difference between TR/BTR and BTR/LTR $(p<0.00004)$ (Table 1).

In maxillary first molars, mean DBTR, MPDCR, and OR were $3 \pm$ $0.34,4.89 \pm 0.56,7.89 \pm 0.91$, respectively. The MPDCR/DBTR (1.62 \pm $0.02)$ and OR/MPDCR $(1.61 \pm 0.0008)$ ratios were in the divine golden ratio. There was statistical difference between the two ratios $(p<$ 0.015). Similarly, DBTR, MPTR, and OR for maxillary second molar were $2.43 \pm 0.43,3.94 \pm 0.67,6.38 \pm 1.1$, respectively. The mean MPDCR/DBTR ratio was $1.62 \pm 0.02$, whereas the mean OR/MPDCR ratio was $1.61 \pm 0.008$. There was no statistically significant difference between TR/BTR and BTR/LTR $(p<0.16)$ (Table 2).

\section{Discussion}

Golden ratio is a geometric proportion that is hypothesized to be most esthetically pleasing to the eye and at the same time most efficient in structure and function. It has been suggested that for appreciation of beauty the human mind functions at the limbic level in attraction to proportions which are in harmony with the golden ratio of 1:1.618. ${ }^{7}$ Many authors have tried to explore the presence of divine ratio in human teeth and to correlate its significance in clinical applications. Levin ${ }^{3}$ was among the first to report that on the frontal view the golden proportion is the most harmonious apparent tooth-to-tooth ratio. It was stated that the observable width of maxillary central and lateral incisors, as the observable widths of maxillary lateral incisors and canines meet the golden ratio. However, others have argued that it is a theoretical proportion and does not necessarily exist in natural dentition. ${ }^{8,9}$ To evaluate this and come to a conclusion regarding the clinical significance of golden ratio in esthetic dentistry, Tridapalli and Steinbach ${ }^{10}$ carried out an integrative study and found the possibility of using the golden ratio as a guide in esthetic rehabilitation as long as the characteristics of each individual are taken into account. Recently, Anand et al. ${ }^{6}$ studied human permanent teeth and identified that the human teeth are divided into two parts at the cementoenamel junction in the golden proportion. It was proposed that such a ratio could have functional implications and hence treatment modalities such as implant placement should exploit the use of this information. In this regard, size of implant can be adjusted as per the divine golden ratio for optimized functional efficiency and success of implant therapy.

In this study, we found that the occlusal anatomy of maxillary and mandibular first and second premolars and that of maxillary first molar follow the divine golden ratio. It was observed that the buccal and lingual/palatal triangular ridges of maxillary and mandibular premolars are divided according to the golden proportion at the central groove. Similarly, the OR of maxillary first molars was found to be proportioned in the divine ratio along the oblique groove. Because of the anatomical variations frequently observed with the third molars, they were not included in the study. The golden ratio has been suggested to represent an instructional pattern that is geometrically encoded within the brain that guides us to recognize beauty. ${ }^{7}$ It is not only esthetically pleasing but is also structurally efficient. In addition to its function in providing an appealing and esthetic smile and aiding in phonetics, an important role of teeth is mastication of food. ${ }^{6}$

A biting force of $120 \mathrm{~kg}$ was recorded by Zhao and $\mathrm{Ye}^{11}$ in one of their earlier work. Thus, it can be believed that the size and shape of the teeth are predetermined and are in harmony with the amount of load they bear. With this in mind, we measured the occlusal table of the posterior teeth and found that the buccal and lingual/palatal triangular ridges of maxillary and mandibular premolars are in golden proportion and the OR of maxillary first molars that is considered as the major stress-bearing structure is also divided by the oblique groove in divine proportion. Since, divine ratios observed in this study were present on occlusal aspect and in posterior teeth, they are the mark of functional efficiency. They might not symbolize the esthetics of the study. Clinicians should incorporate this knowledge of golden proportion while restoring the posterior teeth as 
Golden Ratio in the Ridges of Tooth

Table 1: Golden ratios in transverse ridge of maxillary and mandibular premolars

\begin{tabular}{|c|c|c|c|c|c|c|c|}
\hline Sample & BTR & LTR & $T R$ & TR/BTR & BTR/LTR & $t$ & $p$ value \\
\hline Maxillary first premolar & $4.07 \pm 0.64$ & $2.49 \pm 0.4$ & $6.56 \pm 1.04$ & $1.61 \pm 0.007$ & $1.63 \pm 0.02$ & 5.41 & $<0.00001$ \\
\hline Maxillary second premolar & $4.07 \pm 0.52$ & $2.5 \pm 0.32$ & $6.57 \pm 0.84$ & $1.61 \pm 0.01$ & $1.62 \pm 0.04$ & 0.92 & 0.18 \\
\hline Mandibular first premolar & $2.72 \pm 0.31$ & $1.65 \pm 0.18$ & $4.37 \pm 0.5$ & $1.6 \pm 0.008$ & $1.64 \pm 0.02$ & 6.37 & $<0.00001$ \\
\hline Mandibular second premolar & $3.44 \pm 0.52$ & $2.16 \pm 0.29$ & $5.69 \pm 0.73$ & $1.61 \pm 0.01$ & $1.63 \pm 0.02$ & 4.39 & 0.00004 \\
\hline
\end{tabular}

$\mathrm{TR}$, transverse ridge; $\mathrm{BTR}$, buccal triangular ridge; LTR, lingual triangular ridge

Table 2: Golden ratio in oblique ridge of maxillary first and molars

\begin{tabular}{llllllll}
\hline Sample & MPDCR & DBTR & OR & MPDCR/DBTR & OR/MPDCR & $t$ & $p$ value \\
\hline Maxillary first molar & $4.89 \pm 0.56$ & $3 \pm 0.34$ & $7.89 \pm 0.91$ & $1.62 \pm 0.02$ & $1.61 \pm 0.0008$ & 2.23 & 0.015 \\
Maxillary second molar & $3.94 \pm 0.67$ & $2.43 \pm 0.43$ & $6.38 \pm 1.1$ & $1.62 \pm 0.02$ & $1.61 \pm 0.008$. & 0.99 & 0.16 \\
\hline
\end{tabular}

DBTR, triangular ridge of distobuccal cusp; MPDCR, distal cuspal ridge of mesiopalatal cusp; OR, oblique ridge

it can be a powerful tool in restoring the maximal functional efficiency of the tooth. This concept can also be integrated in fixed prosthodontics during the designing and fabrication of prosthetic crowns and bridges. Golden proportion also holds a place of importance in restorative dentistry. Restorative dentistry loses its significance in the absence of good occlusion. Occlusion is not just about teeth but it demands a balance and harmony of the entire stomatognathic system. ${ }^{12,13}$ Thus, it is essential to not only take into consideration the interrelationship between teeth and the adjacent anatomical structures but importance should also be given to the minute anatomical feature of the concerned tooth as well such as the relative proportion of triangular ridge or OR. ${ }^{14}$ Attempts should be made at restoring the anatomy of lost tooth structure in the best possible ways so as to re-establish the original functional efficiency of the tooth. Among the various other factors, a clinician should implement the concept of golden proportion for the same.

Dental casts were used in this study for obtaining the samples. There is a possibility of incorporation of minor errors in the measurements, as cast does not represent the exact replica of the natural structure present in the oral cavity. Extracted tooth could be a good option for such studies but it is very difficult to obtain such specimens with unaltered morphology. However, the present method of measurement was the best possible way to obtain the exact values.

In this study, only orthodontic casts were selected for analysis. Hence, the age of the patients was mostly in the range of 18-25 years. This age range is highly suitable for such kind of studies where normal anatomical structures are studied for their measurements. There are less post eruptive regressive changes in the morphology of tooth in this age group, thus reducing the errors in obtaining the golden ratio. Since minute errors in the measurements are very critical in such studies, outmost care in terms of inclusion and exclusion criteria was instituted while selection of the casts for analysis. Third molars showed wide degree of morphological variations ${ }^{15}$ and hence it is not included in this study for finding the golden ratios.

\section{Conclusion}

In conclusion, the ORs of maxillary first molar ridge and TR of maxillary and mandibular premolars are divided into two parts at transverse and central grooves, respectively, in a divine ratio. This signifies the strength and stability of the occlusal table in the posterior teeth, as the golden ratio is the mark of functional excellence. Golden ratio should be taken into consideration while restoration or fabrication of the posterior teeth. However, future in vitro and in vivo studies are recommended to assess the functional superiority of those occlusal rides in the golden ratio. We also recommend studies on finding other possible structure that could be present in the golden ratio on the tooth surface as well as at the microscopic level.

\section{References}

1. Borisavljević M. The Golden Number. London: Alec Tiranti; 1958.

2. Gradner M. More Mathematical Puzzles. London: Penguin Books; 1966.

3. Levin El. Dental esthetics and the golden proportion. J Prosthet Dent 1978;40(3):244-252. DOI: 10.1016/0022-3913(78)90028-8.

4. Reddy TVK, Dhanasekarna S. The golden proportion. J Oper Dent Endod 2017;2(2):79-83. DOI: 10.5005/jp-journals-10047-0042.

5. Elam K. Geometry of design: studies in proportion and composition. New York: Princeton Architectural Press; 2001.

6. Anand R, Sarode SC, Sarode GS, et al. Human permanent teeth are divided into two parts at the cemento-enamel junction in the divine golden ratio. Indian J Dent Res 2017;28(6):609-612. DOI: 10.4103/ijdr. IJDR_525_16.

7. Laxmikanth SM, Raghavendra SR. Golden proportion: a review. J Adv Clin Res Insights 2014;1(1):25-29. DOI: 10.15713/ins.jcri.8.

8. Mahshid M, Khoshvaghti A, Varshosaz M, et al. Evaluation of golden proportion in individuals with an esthetic smile. J Esthet Restor Dent 2004;16(3):185-192. DOI: 10.1111/j.1708-8240.2004.tb00032.x.

9. Sandeep N, Satwalekar P, Srinivas S, et al. An analysis of maxillary anterior teeth dimensions for the existence of golden proportion: clinical study. J Int Oral Health 2015;7(9):18-21.

10. Tridapalli LP, Steinbach M. The use of golden proportion in dentistry: a integrative review. Rev Odonto Ciencia 2018;33(1):77-83. DOI: 10.15448/1980-6523.2018.1.29703.

11. Zhao $Y, Y e D$. Measurement of biting force of normal teeth at different ages. J West China Univ Med Sci 1994;25(4):414-417.

12. Sandhu S, Lal J, Singh R, et al. Significance of establishing occlusal anatomy in operative dentistry. Saint Int Dent J 2016;2(1):7-10. DOI: 10.4103/2454-3160.202121.

13. Sarode GS, Sarode SC. Abfraction: a review. J Oral Maxillofac Pathol 2013;17(2):222-227. DOI: 10.4103/0973-029X.119788.

14. Hess TA, Wadhwani CP. The Tucker technique: conservative molar inlays preserving the transverse ridge. Oper Dent 2012;37(1):93-97. DOI: 10.2341/11-048-T.

15. Trinks PW, Grifo MB, Pari F, et al. Characterization of third molar morphometric variables. Acta Odontol Latinoam 2016;29(2): $144-150$. 